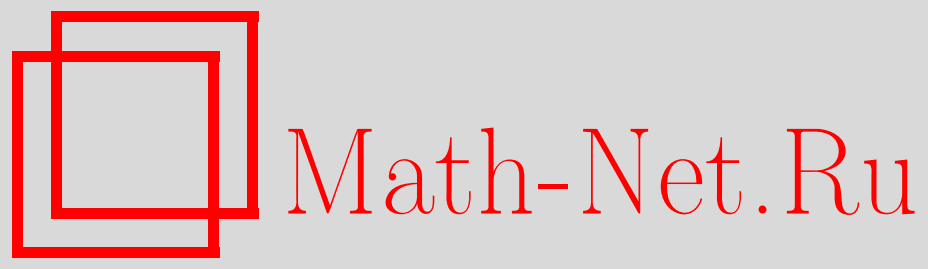

М. С. Агранович, Смешанные задачи в липшицевой области для сильно эллиптических систем 2-го порядка, Функи. анализ и его прил., 2011, том 45, выпуск 2, 1-22

DOI: https://doi.org/10.4213/faa3039

Использование Общероссийского математического портала MathNet.Ru подразумевает, что вы прочитали и согласны с пользовательским соглашением http://www . mathnet.ru/rus/agreement

Параметры загрузки:

IP : 54.80 .97 .219

26 апреля 2023 г., 13:04:48

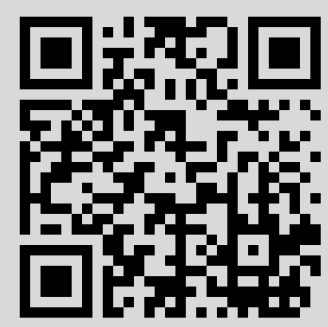




\title{
Смешанные задачи в липшицевой области для сильно эллиптических систем 2-го порядка*
}

\author{
(c) 2011. М. С. АГРАНович
}

\begin{abstract}
Рассматриваются смешанные задачи для сильно эллиптических систем 2-го порядка в ограниченной области пространства $\mathbb{R}^{n}$ с липшицевой границей. Выводятся уравнения на границе, эквивалентные задаче, в простейших $L_{2}$-пространствах $H^{s}$ типа Соболева, что позволяет представить решения через поверхностные потенциалы. Доказывается результат о регулярности решений с выходом в немного более общие пространства $H_{p}^{s}$ бесселевых потенциалов и пространства $B_{p}^{s}$ Бесова. Рассматриваются задачи со спектральным параметром в системе или в условии на части границы, обсуждаются спектральные свойства соответствующих операторов, включая асимптотики собственных значений.
\end{abstract}

\section{§1. Введение}

1.1. Содержание работы. Пусть $\Omega$ - ограниченная область в пространстве $\mathbb{R}^{n}, n \geqslant 2$, с липшицевой границей $Г$. Пусть в $\Omega$ задана сильно эллиптическая система 2-го порядка $L u=f$, записанная в дивергентной форме, см. ниже (1.1). Предположения о гладкости коэффициентов в известной мере минимизируются. Основную «энергетическую» форму $\Phi_{\Omega}(u, v)$ мы предположим коэрцитивной при $u=v$ в усиленном смысле на пространстве $H^{1}(\Omega)=W_{2}^{1}(\Omega)$; это условие влечет однозначную разрешимость задач Дирихле и Неймана.

В бо́льшей части работы (§§1-6) мы предполагаем, что граница Г разбита на две области $\Gamma_{1}$ и $\Gamma_{2}$ замкнутой $(n-1)$-мерной липшицевой поверхностью $\partial \Gamma_{j}$ без самопересечений, на $\Gamma_{1}$ ставится условие Дирихле, на $\Gamma_{2}-$ Неймана.

Смешанным задачам посвящена обширная литература. Причина состоит в том, что они встречаются во множестве прикладных вопросов. См., например, книги [16] и [41]. (В них содержатся также явные и непростые формулы для решения ряда конкретных смешанных задач.)

Во многих работах накладываются дополнительные условия гладкости на $\Gamma_{1}$ и $\Gamma_{2}$, или на $\Gamma$, и на $\partial \Gamma_{j}$. См., в частности, [39], [38] и приведенную там литературу, а также [42], [43], [30]. В ряде работ накладывается условие на геометрию границы: обычно требуется, грубо говоря, чтобы угол между нормалями к $\Gamma_{1}$ и $\Gamma_{2}$ в точках на $\partial \Gamma_{j}$ был меньше $\pi$ (creased domains, точное определение см. в [10]; см. также [46], [27], [11]). Многие работы посвящены конкретным уравнениям или системам, чаще всего это уравнения Лапласа-Гельмгольца или системы теории упругости; кроме названных выше работ, см. также [19], [35]. При этом исследования в значительной степени направлены на выяснение регулярности решения и в особенности его поведения вблизи $\partial \Gamma_{j}$. Легко строятся примеры (типа $r^{\alpha} \sin (\alpha \theta)$ в полярных координатах $r, \theta$ в плоской области в случае уравнения Лапласа), показывающие, что даже при бесконечно гладких $\Gamma_{j}$ (или

\footnotetext{
*Поддержано грантом РФФИ 11-01-00277-а.
} 
даже $Г)$ и $\partial \Gamma_{j}$ решение может иметь особенность вблизи $\partial \Gamma_{j}$. В [42], [43], [30] в гладком случае получена асимптотика решения вблизи $\partial \Gamma_{j}$ при помощи метода Винера-Хопфа в форме, предложенной в [15].

Существенно меньше работ об общих сильно эллиптических уравнениях или системах, в которых никаких дополнительных условий гладкости на $\Gamma_{j}$ и $\partial \Gamma_{j}$ или геометрических условий не накладывается. Назовем монографию [24], работы [34] (о скалярном уравнении с формально самосопряженной старшей частью), [18] и [33, п. 3] (о скалярном формально самосопряженном уравнении без младших членов). K этому направлению относится и наша работа (в пределах $\S \S 1-5)$.

Мы приведем общую теорему о существовании и единственности вариационного решения в пространствах $H^{s}$ и сравним разные подходы к ее доказательству. С учетом деликатности некоторых из затрагиваемых вопросов мы сначала напомним свойства пространств $H^{s}$ (п. 1.3), постановку задач Дирихле и Неймана (п. 2.1) и непосредственное доказательство однозначной разрешимости смешанной задачи на основе леммы Лакса-Мильграма (п. 2.2). Затем, в 33 , выведем двумя способами уравнения на Г с операторами типа поверхностных потенциалов на части границы, эквивалентные задаче и однозначно разрешимые. Эти операторы представляют самостоятельный интерес. В [24] уравнения получены в предположении формальной самосопряженности старшей части системы. Нам оно не понадобится; мы воспользуемся прозрачным подходом в [30] к системе анизотропной упругости.

В $\S 4$ мы обобщим теорему об однозначной разрешимости на близкие к $H^{s}$ пространства $H_{p}^{s}$ бесселевых потенциалов и пространства $B_{p}^{s}$ Бесова, используя теорему Шнейберга [40] об экстраполяции обратимости операторов, действующих в интерполяционных шкалах пространств. Ср., в частности, [27] (где получены очень общие теоремы об однозначной разрешимости для уравнения Лапласа при дополнительном геометрическом предположении), а также [11]. Отметим, что в этих работах и ряде других авторы выходят за рамки функциональных пространств, используемых в нашей статье.

В $\S 5-6$ мы рассмотрим спектральные задачи. Для самосопряженных задач мы укажем результаты о базисности собственных функций, а для несамосопряженных задач и задач в рассматриваемых у нас негильбертовых пространствах - условия полноты корневых функций и суммируемости рядов Фурье по ним методом Абеля-Лидского, ср. [3]-[5].

Если система или хотя бы ее старшая часть формально самосопряженная, то интересна асимптотика собственных значений. Для задач со спектральным параметром в системе результат следует из теоремы в [25], и там получена сильная оценка остаточного члена. Задачи со спектральным параметром в условии на части границы - это спектральные задачи Пуанкаре-Стеклова. Мы применим к ним вариационный подход, восходящий к Куранту-Гильберту [13], при условии, что граница почти гладкая (гладкая вне замкнутого подмножества $\Gamma_{\text {sing }}$ нулевой меры, это определение из [6]). Мы дополним также результаты по спектральным асимптотикам, полученные в [4] и [5].

В $\S 7$ мы кратко рассмотрим некоторые обобщения.

1.2. Уточнение постановки задачи. Запишем систему в виде

$$
L u:=-\sum \partial_{j} a_{j, k}(x) \partial_{k} u(x)+\sum b_{j}(x) \partial_{j} u(x)+c(x) u(x)=f(x) .
$$


Здесь и дальше $\partial_{k}=\partial / \partial x_{k} ; u$ - вектор-функция (столбец) размерности $m$, так что коэффициенты - это $m \times m$-матрицы. Как и $u$, они состоят из комплекснозначных функций. Будем предполагать, что $a_{j, k} \in C^{1}(\bar{\Omega}), b_{j} \in C^{0,1}(\bar{\Omega})$ (липшицевы), $c \in L_{\infty}(\Omega)$. В некоторых ситуациях можно предполагать меньше. Условие сильной эллиптичности состоит в равномерной положительной определенности вещественной части $\frac{1}{2}\left(a+a^{*}\right)$ главного символа - матрицы $a(x, \xi)=\sum a_{j, k}(x) \xi_{j} \xi_{k}$ - при вещественных $\xi,|\xi|=1$. Решение будем сначала искать в $H^{1}(\Omega)$. Пространство для $f$ будет указано в п. 2.2 .

Запишем граничные условия:

$$
u^{+}=g \quad \text { на } \Gamma_{1}, \quad T^{+} u=h \quad \text { на } \Gamma_{2} .
$$

Здесь $u^{+}=g$ - след функции $u$, принадлежащий $H^{1 / 2}\left(\Gamma_{1}\right)$, мы его будем обозначать также через $\gamma^{+} u$, а $T^{+} u=h$ - конормальная производная, ее определение напоминается и кратко комментируется в п. 2.1; она принадлежит $H^{-1 / 2}\left(\Gamma_{2}\right)$.

Системе отвечает обозначаемая через $\Phi_{\Omega}(u, v)$ полуторалинейная форма

$$
\int_{\Omega}\left[\sum a_{j, k}(x) \partial_{k} u(x) \cdot \partial_{j} \bar{v}(x)+\sum b_{j}(x) u(x) \cdot \bar{v}(x)+c(x) u(x) \cdot \bar{v}(x)\right] d x ;
$$

она определена на функциях из $H^{1}(\Omega)$. Наше предположение о ее (усиленной) коэрцитивности состоит в том, что для них справедливо неравенство Гординга

$$
\|u\|_{H^{1}(\Omega)}^{2} \leqslant C_{0} \operatorname{Re} \Phi_{\Omega}(u, u)
$$

без добавления $\|u\|_{L_{2}(\Omega)}^{2}$ справа. На функциях с $u^{+}=0$ на $Г$ такое неравенство является следствием одной лишь сильной эллиптичности, если

$$
\operatorname{Re}(c u, u)_{\Omega} \geqslant C_{1}\|u\|_{L_{2}(\Omega)}^{2}
$$

с достаточно большой постоянной $C_{1}$, что у нас предполагается. Здесь $(\cdot, \cdot)_{\Omega}$ - стандартное скалярное произведение в $L_{2}(\Omega)$. На функциях из $H^{1}(\Omega)$ неравенство (1.4) получается в случае скалярного уравнения с вещественной симметрической матрицей из старших коэффициентов. Для его справедливости в матричном случае на коэффициенты в $L$ накладываются некоторые (достаточные) условия (см., например, [24], [5]); в частности, они выполнены для обобщенных систем теории упругости (см., например, [32]). При этом снова форма $\operatorname{Re}(c u, u)_{\Omega}$ считается достаточно большой.

1.3. Пространства $H^{s}$. См., например, [24]. Пространства $H^{s}\left(\mathbb{R}^{n}\right)$ бесселевых потенциалов $(s \in \mathbb{R})$ вводятся формулой

$$
H^{s}\left(\mathbb{R}^{n}\right)=J^{-s} L_{2}\left(\mathbb{R}^{n}\right), \quad J^{-s}=F^{-1}(1+|\xi|)^{-s} F,
$$

где $F$ - преобразование Фурье в смысле обобщенных функций; $\|u\|_{H^{s}\left(\mathbb{R}^{n}\right)}=$ $\left\|J^{s} u\right\|_{L_{2}\left(\mathbb{R}^{n}\right)}$. Для $s \geqslant 0$ это пространства $W_{2}^{s}\left(\mathbb{R}^{n}\right)$ Соболева (при целых $s$ ) и Слободецкого (при нецелых $s)$. Пространства $H^{s}\left(\mathbb{R}^{n}\right)$ и $H^{-s}\left(\mathbb{R}^{n}\right)$ дуальны, или двойственны, или сопряжены, относительно продолжения стандартного скалярного произведения в $L_{2}\left(\mathbb{R}^{n}\right)$ на их прямое произведение.

Пространство $H^{s}(\Omega)$ определяется как состоящее из сужений элементов из $H^{s}\left(\mathbb{R}^{n}\right)$ на $\Omega$ в смысле обобщенных функций с обычной нормой inf. Имеется универсальный ограниченный оператор $\mathscr{E}$ продолжения элементов из $H^{s}(\Omega)$ до элементов из $H^{s}\left(\mathbb{R}^{n}\right)$, не зависящий от $s$ [37]. 
Пространство $\widetilde{H}^{s}(\Omega)$ определяется как подпространство в $H^{s}\left(\mathbb{R}^{n}\right)$ элементов с носителями в $\bar{\Omega}$. Норма наследуется из $H^{s}\left(\mathbb{R}^{n}\right)$. Пространство $\widetilde{H}^{s}(\Omega)$ можно отождествить с пополнением в $H^{s}\left(\mathbb{R}^{n}\right)$ линеала $C_{0}^{\infty}(\Omega)$, считая функции из него продолженными нулем вне $\Omega$. При $-1 / 2<s<3 / 2, s \neq 1 / 2$ его можно отождествить также с пополнением $\stackrel{\circ}{H}^{s}(\Omega)$ линеала $C_{0}^{\infty}(\Omega)$ в $H^{s}(\Omega)$. Все отождествления понимаются с точностью до эквивалентности норм.

Пространства $\widetilde{H}^{-s}(\Omega)$ и $H^{s}(\Omega)$ дуальны относительно продолжения стандартного скалярного произведения в $L_{2}(\Omega)$ на их прямое произведение. Это продолжение имеет вид

$$
(f, v)_{\Omega}=(f, \mathscr{E} v)_{\mathbb{R}^{n}}
$$

где справа используется продолжение скалярного произведения в $L_{2}\left(\mathbb{R}^{n}\right)$. Рaзумеется, функции в этих формах до и после запятой можно одновременно поменять местами. Пространства $H^{s}(\Omega)$ и $\widetilde{H}^{s}(\Omega)$ отождествляются при $|s|<1 / 2$.

Пространства $H^{s}(\Gamma)$ определяются на липшицевой поверхности при $|s| \leqslant 1$ при помощи разбиения единицы и норм в $H^{s}\left(\mathbb{R}^{n-1}\right)$. Оператор $\gamma^{+} v=v^{+}$перехода к следу действует ограниченным образом из $H^{s+1 / 2}(\Omega)$ в $H^{s}(\Gamma)$ при $0<s<1$ и имеет ограниченный правый обратный. Пространства $H^{s}(\Gamma)$ и $H^{-s}(\Gamma)$ дуальны относительно продолжения стандартного скалярного произведения в $L_{2}(\Gamma)$ (относительно естественно определяемой меры - площади поверхности на $\Gamma$ ) на их прямое произведение.

Пространство $\widetilde{H}^{-s}(\Omega)$ при $s>1 / 2$ содержит элементы из $H^{-s}\left(\mathbb{R}^{n}\right)$, сосредоточенные на $Г$. При $1 / 2<s<3 / 2$ они имеют вид $\left(w, v^{+}\right)_{\Gamma}, v \in H^{s}(\Omega)$, $w \in H^{-s+1 / 2}(\Gamma)[26]$.

Если $\Gamma_{0}$ - область на $\Gamma$ с липшицевой границей, то пространство $H^{s}\left(\Gamma_{0}\right)$ определяется при $|s| \leqslant 1$ как состоящее из сужений на $\Gamma_{0}$ элементов из $H^{s}(\Gamma)$ с нормой inf. Имеется ограниченный оператор продолжения из $H^{s}\left(\Gamma_{0}\right)$ в $H^{s}(\Gamma)$, не зависящий от $s$. Пространство $\widetilde{H}^{s}\left(\Gamma_{0}\right)$ определяется как подпространство в $H^{s}(\Gamma)$ элементов с носителями в $\bar{\Gamma}_{0}$. Пространства $\widetilde{H}^{-s}\left(\Gamma_{0}\right)$ и $H^{s}\left(\Gamma_{0}\right)$ дуальны относительно продолжения стандартного скалярного произведения в $L_{2}\left(\Gamma_{0}\right)$ на их прямое произведение. Это продолжение строится аналогично (1.7). Пространства $H^{s}\left(\Gamma_{0}\right)$ и $\widetilde{H}^{s}\left(\Gamma_{0}\right)$ отождествляются при $|s|<1 / 2$.

Оператор умножения на характеристическую функцию области $\Gamma_{0}$ является мультипликатором в $H^{s}(\Gamma)$ при $|s|<1 / 2$ и не является мультипликатором в $H^{ \pm 1 / 2}(\Gamma)$. Но он является мультипликатором в $\widetilde{H}^{s}\left(\Gamma_{0}\right)$ при $|s| \leqslant 1 / 2 ;$ более того, умножение на эту функцию не меняет элементы этих пространств.

\section{§2. Вариационный подход}

2.1. Задачи Дирихле и Неймана. Напомним их вариационную постановку. Запишем формулу Грина

$$
\Phi_{\Omega}(u, v)=(L u, v)_{\Omega}+\left(T^{+} u, v^{+}\right)_{\Gamma} .
$$

Здесь $v-$ произвольная пробная функция.

Начнем с задачи Неймана $\left(\Gamma=\Gamma_{2}\right)$. В этом случае $u, v \in H^{1}(\Omega)$, так что след $\gamma^{+} v=v^{+}$принадлежит $H^{1 / 2}(\Gamma)$. В соответствии с выбором пространства для $v$ правая часть системы $L u=f$ берется из $\widetilde{H}^{-1}(\Omega)$. Справа в $(2.1)$ используются соответствующие дуальности. 
Конормальная производная на гладких функциях определяется формулой $T^{+} u(x)=\sum \nu_{j}(x) \partial_{j} u(x)$ в точках $x$ границы, в которых есть нормаль к ней (это почти все точки). Здесь $\nu_{j}(x)$ - коэффициенты единичной внешней нормали. В общем случае заданием функции $u \in H^{1}(\Omega)$ обобщенная функция $L u(x)$ однозначно определяется только внутри области $\Omega$, а $f \in \widetilde{H}^{-1}(\Omega)$ может содержать еще слагаемое из $H^{-1}\left(\mathbb{R}^{n}\right)$ с носителем на $\Gamma$. Справедливость формулы (2.1) постулируется, и она принимается за определение конормальной производной $T^{+} u \in H^{-1 / 2}(\Gamma)$ (по $u$ и $L u=f$ ), а также за определение решения задачи Неймана (по $f=L u$ и $h=T^{+} u$ ). Всегда можно принять, что $h=0$, изменив $f$ на слагаемое с носителем на $\Gamma$.

Из неравенства (1.4) следует однозначная разрешимость этой задачи в силу следующей леммы Лакса-Мильграма о слабых решениях абстрактного уравнения $L u=f$, где $L-$ ограниченный оператор, определяемый приведенным ниже равенством (2.4). (Ср., например, [24, с. 43].)

Лемма 2.1. Пусть $H$ - гильбертово пространство и $H^{*}-$ сопряженное $\kappa$ нему пространство относительно формы $(f, v), v \in H, f \in H^{*}$, и пусть задано $f \in H^{*}$. Предположим, что для непрерывной полуторалинейной формы $\Phi(u, v)$ на $H$ выполнено неравенство

$$
\|u\|_{H}^{2} \leqslant C \operatorname{Re} \Phi_{\Omega}(u, u) .
$$

Тогда существует один и только один элемент $u \in H$, такой, что

$$
\Phi(u, v)=(f, v)
$$

при всех $v \in H$. При этом оператор $L^{-1}: f \mapsto u$ ограничен.

Перейдем к задаче Дирихле $\left(\Gamma=\Gamma_{1}\right)$. В этом случае $u \in H^{1}(\Omega)$ и $v \in \widetilde{H}^{1}(\Omega)$. Соответственно $f \in H^{-1}(\Omega)$. Формула Грина принимает вид

$$
\Phi_{\Omega}(u, v)=(L u, v)_{\Omega} .
$$

В эту формулу $u^{+}$не входит явным образом, но подразумевается, что $u^{+}=g$. Пусть $u_{0}-$ функция из $H^{1}(\Omega)$ с $u_{0}^{+}=g$. Определим $f_{0}=L u_{0} \in H^{-1}(\Omega)$ формулой Грина $\Phi_{\Omega}\left(u_{0}, v\right)=\left(L u_{0}, v\right)_{\Omega}$. Разность $u-u_{0}$ принадлежит $\widetilde{H}^{1}(\Omega)=$ $\stackrel{\circ}{H}^{1}(\Omega)$, и если ее переобозначить через $u$, то снова получается формула Грина (2.4), в которой уже обе функции $u$ и $v$ принадлежат $\widetilde{H}^{1}(\Omega)$, а $f=L u-$ сопряженному пространству $H^{-1}(\Omega)$. Это стандартная постановка задачи Дирихле с однородным граничным условием. Форма $\Phi_{\Omega}(u, u)$ у нас коэрцитивна на $\widetilde{H}^{1}(\Omega)$, так что задача Дирихле однозначно разрешима.

Замечание. Предположим (в этом замечании), что оператор $L-$ формально самосопряженный. Из формулы Грина видно, что тогда пространство $H^{1}(\Omega)$ есть ортогональная сумма относительно скалярного произведения $\Phi_{\Omega}(u, v)$ подпространства $\stackrel{\circ}{H}^{1}(\Omega)=\widetilde{H}^{1}(\Omega)$ и подпространства решений системы $L u=0$. Последнее параметризуется данными Дирихле в $H^{1 / 2}(\Gamma)$, поэтому линейные непрерывные функционалы на нем можно привести в изоморфное соответствие с функционалами из $H^{-1 / 2}(\Gamma)$ над $H^{1 / 2}(\Gamma)$. По этим причинам пространство $H^{-1}(\Omega)$, дуальное к $\widetilde{H}^{1}(S)$, можно отождествить с фактор-пространством пространства $\widetilde{H}^{-1}(\Omega)$, дуального к $H^{1}(\Omega)$, по подпространству функционалов, сосредоточенных на $\Gamma$. 
2.2. Перейдем к смешанной задаче. Ее решения $u$ тоже ищутся в $H^{1}(\Omega)$. Ключевым моментом можно считать выбор пространства пробных функций $v$ : это (замкнутое) подпространство $H^{1}\left(\Omega, \Gamma_{1}\right)$ в $H^{1}(\Omega)$, состоящее из функций с нулевым следом на $\Gamma_{1}$. (То есть след, принадлежащий $H^{1 / 2}(\Gamma)$, равен 0 на $\Gamma_{1}$ ).

Как видно из [27], пространство $H^{1}\left(\Omega, \Gamma_{1}\right)$ можно определить также как 1 ) пространство сужений на $\Omega$ элементов пополнения линеала $C_{0}^{\infty}\left(\mathbb{R}^{n} \backslash \bar{\Gamma}_{1}\right)$ в $\left.H^{1}\left(\mathbb{R}^{n}\right), 2\right)$ пополнение в $H^{1}(\Omega)$ сужений на $\Omega$ функций из $C_{0}^{\infty}\left(\mathbb{R}^{n} \backslash \bar{\Gamma}_{1}\right)$.

Формула Грина, определяющая решение задачи, принимает вид

$$
\Phi_{\Omega}(u, v)=(L u, v)_{\Omega}+\left(T^{+} u, v^{+}\right)_{\Gamma_{2}},
$$

где $L u=f$ и $T^{+} u=h$. Выбором пространства для $v$ определяется пространство правых частей системы: это пространство

$$
\widetilde{H}^{-1}\left(\Omega, \Gamma_{1}\right):=\left[H^{1}\left(\Omega, \Gamma_{1}\right)\right]^{*},
$$

сопряженное к $H^{1}\left(\Omega, \Gamma_{1}\right)$ относительно продолжения формы $(f, v)_{\Omega}$ на их прямое произведение. По аналогии с замечанием в конце п. 2.1 получается, что (2.6) можно отождествить с фактор-пространством пространства $\widetilde{H}^{-1}(\Omega)$ по подпространству функционалов, сосредоточенных на $\bar{\Gamma}_{1}$. Тогда любое $f$ из $(2.6)-$ элемент из $\widetilde{H}^{-1}(\Omega)$, определенный с точностью до прибавления любого элемента с носителем на $\bar{\Gamma}_{1}$. Можно просто считать $f$ элементом пространства $\widetilde{H}^{-1}(\Omega)$ (продолжая соответствующий функционал на все $H^{1}(\Omega)$ по теореме Хана-Банаха), фактически это и сделано в [24].

Снова равенство $g=u^{+}$подразумевается. Возьмем функцию $u_{0}$ из $H^{1}(\Omega)$ с $u_{0}^{+}=g$ на $\Gamma_{1}$ (продолжим $g$ до функции из $H^{1 / 2}(\Gamma)$ и возьмем $u_{0}$ с $u_{0}^{+}=g$ ). Определим $L u_{0}$ формулой $\Phi_{\Omega}\left(u_{0}, v\right)=\left(L u_{0}, v\right)_{\Omega}$, принимая соответствующую конормальную производную равной нулю на $\Gamma_{2}$. Переобозначим разность $u-u_{0}$ через $u$. Для нее получается формула Грина (2.5), в которой обе функции $u$ и $v$ принадлежат пространству $H^{1}\left(\Omega, \Gamma_{1}\right)$, а $L u=f$ принадлежит сопряженному пространству (2.6). При желании можно принять, что $h=0$.

Все это согласуется со сказанным выше про задачи Дирихле и Неймана.

Теперь, используя коэрцитивность формы $\Phi_{\Omega}(u, u)$ на $H^{1}\left(\Omega, \Gamma_{1}\right)$ (ее сейчас достаточно), при помощи леммы Лакса-Мильграма сразу получаем следующую известную теорему. Сp. [24].

Теорема 2.2. При любых $f \in \widetilde{H}^{-1}\left(\Omega, \Gamma_{1}\right), g \in H^{1 / 2}\left(\Gamma_{1}\right), h \in H^{-1 / 2}\left(\Gamma_{2}\right)$ задача (1.1)-(1.2) имеет одно и только одно вариачионное решение $и \in H^{1}(\Omega)$.

\section{§3. Сведе́ние смешанной задачи к уравнениям на $Г$}

3.1. Для экономии места предположим, что область $\Omega=\Omega^{+}$лежит на стандартном торе $\mathbb{T}^{n}$ с периодическими координатами и поверхность $\Gamma$ разделяет его на две области $\Omega^{ \pm}$. Нормаль к $\Gamma$ в тех точках, где она есть, считаем направленной в $\Omega^{-}$. Пространство $\widetilde{H}^{s}\left(\Omega^{+}\right)$теперь будет подпространством в $H^{s}(\mathbb{T})$. Коэффициенты системы считаем продолженными на тор с сохранением предположений об их «гладкости» и сильной эллиптичности. Форму $\Phi_{\mathbb{T}}(u, u)$ предположим коэрцитивной (в усиленном смысле) на $H^{1}(\mathbb{T})$ (для этого не требуются дополнительные предположения о старшей части системы), а формы 
$\Phi_{\Omega^{ \pm}}(u, u)$ - на $H^{1}\left(\Omega^{ \pm}\right)$. Тогда задачи Дирихле и Неймана в $\Omega^{ \pm}$однозначно разрешимы. Это позволяет воспользоваться готовыми результатами из [3] (где мы следовали идеям из [31], [12] и [24]). Мы продолжим рассмотрение смешанной задачи в $\Omega=\Omega^{+}$, хотя можно одновременно рассматривать ее и в $\Omega^{-}$.

Система (1.1) у нас однозначно разрешима в $H^{1}(\mathbb{T})$ при $f \in H^{-1}(\mathbb{T})$. (Это позволит в дальнейшем считать, что $f=0$.) Обратный оператор $L^{-1}$ - интегральный:

$$
L^{-1} f(x)=\int_{\mathbb{T}} \mathscr{E}(x, y) f(y) d y .
$$

Это так называемый ньютонов потенциал. Его ядро $\mathscr{E}-$ фундаментальное решение для $L$. Имея его, составим потенииал простого слоя

$$
\mathscr{A} \psi(x)=\int_{\Gamma} \mathscr{E}(x, y) \psi(y) d S_{y}
$$

и потенциал двойного слоя; последний в общем случае определяется формулой

$$
\mathscr{B} \varphi(x)=\int_{\Gamma}\left(\widetilde{T}_{y}^{+} \mathscr{E}^{*}(x, y)\right)^{*} \varphi(y) d S_{y} \quad(x \notin \Gamma),
$$

где $\widetilde{T}^{+}(\cdot)$ - конормальная производная, отвечающая формально сопряженному к $L$ оператору $\widetilde{L}$ (см. [24]). При $L=-\Delta$ звездочки и волна опускаются. Напомним свойства этих операторов (см. [24] и [3]).

При наших предположениях оператор $\mathscr{A}$ продолжается до оператора, действующего ограниченным образом из $H^{-1 / 2}(\Gamma)$ в $H^{1}(\mathbb{T})$ и, значит, в $H^{1}\left(\Omega^{ \pm}\right)$. При $\psi \in H^{-1 / 2}(\Gamma)$ функция $u=\mathscr{A} \psi$ удовлетворяет однородной системе $L u=0$ в $\Omega^{ \pm}$и имеет одинаковые следы $\gamma^{ \pm} u$ на $\Gamma$, обозначаемые через $A \psi$; это ограниченный обратимый оператор из $H^{-1 / 2}(\Gamma)$ в $H^{1 / 2}(\Gamma)$. Это позволяет строить решение задачи Дирихле в $\Omega^{+}$для системы $L u=0$ по формуле

$$
u=\mathscr{A} A^{-1} u^{+} .
$$

Оператор $\mathscr{B}$ действует ограниченным образом из $H^{1 / 2}(\Gamma)$ в $H^{1}\left(\Omega^{ \pm}\right)$, и при $\varphi \in H^{1 / 2}(\Gamma)$ функция $u=\mathscr{B} \varphi$ тоже удовлетворяет однородной системе в $\Omega^{ \pm}$. Она имеет следы $\gamma^{ \pm} \mathscr{B} \varphi$ на $Г$, и это ограниченные операторы в $H^{1 / 2}(\Gamma)$. Положим, как в [24], $B=\frac{1}{2}\left(\gamma^{+} \mathscr{B}+\gamma^{-} \mathscr{B}\right)$. Это так называемое прямое значение потенциала двойного слоя. Скачок $[u]=u^{-}-u^{+}$функции $u=\mathscr{B} \varphi$ равен $\varphi$, поэтому $\gamma^{+} \mathscr{B}=-\frac{1}{2} I+B$. Оператор справа ограничен и обратим в $H^{1 / 2}(\Gamma)$. Это позволяет записать решение задачи Дирихле в $\Omega^{+}$также в виде

$$
u=\mathscr{B}\left(-\frac{1}{2} I+B\right)^{-1} u^{+} .
$$

Операторы $T^{ \pm}$применимы к функциям $\mathscr{A} \psi$ и $\mathscr{B} \varphi$. При этом $T^{+} \mathscr{B}=T^{-} \mathscr{B}$. Оператор $H=-T^{ \pm} \mathscr{B}$ называется гиперсингулярным оператором. Он действует ограниченным образом из $H^{1 / 2}(\Gamma)$ в $H^{-1 / 2}(\Gamma)$ и обратим. Поэтому решение задачи Неймана для системы $L u=0$ в $\Omega^{+}$можно строить по формуле

$$
u=-\mathscr{B} H^{-1} T^{+} u \text {. }
$$

Далее, $T^{ \pm} \mathscr{A}-$ ограниченные операторы в $H^{-1}(\Gamma)$. Положим, как в [24], $\widehat{B}=$ $\frac{1}{2}\left(T^{+} \mathscr{A}+T^{-} \mathscr{A}\right)$. Скачок $[T \mathscr{A} \varphi]=T^{-} \mathscr{A} \varphi-T^{+} \mathscr{A} \varphi$ равен $-\varphi$, поэтому $T^{+} \mathscr{A}=$ 
$\frac{1}{2} I+\widehat{B}$. Это ограниченный обратимый оператор в $H^{-1 / 2}(\Gamma)$, и решение задачи Неймана в $\Omega^{+}$можно строить также по формуле

$$
u=\mathscr{A}\left(\frac{1}{2} I+\widehat{B}\right)^{-1} T^{+} u .
$$

Нам еще нужны оператор $N$ (Neumann-to-Dirichlet), переводящий данные Неймана $T^{+} u$ в данные Дирихле $u^{+}$, и обратный оператор $D$ (Dirichlet-toNeumann). Оператор $N$ действует ограниченным образом из $H^{-1 / 2}(\Gamma)$ в $H^{1 / 2}(\Gamma)$, оператор $D-$ в обратную сторону. Для них справедливы неравенства типа Гординга, вытекающие из формулы Грина (2.1) и исходного предположения о коэрцитивности:

$$
\|\varphi\|_{H^{1 / 2}(\Gamma)}^{2} \leqslant C_{1} \operatorname{Re}(D \varphi, \varphi)_{\Gamma}, \quad\|\psi\|_{H^{-1 / 2}(\Gamma)}^{2} \leqslant C_{2} \operatorname{Re}(N \psi, \psi)_{\Gamma} .
$$

Обратимость операторов $D$ и $N$ следует из предположения об однозначной разрешимости задач Дирихле и Неймана. Она следует также из этих неравенств в силу леммы Лакса-Мильграма. Кроме того, известно, что $B A=A \widehat{B}$, поэтому из написанных выше формул следует, что

$$
N=A\left(\frac{1}{2} I+\widehat{B}\right)^{-1}=\left(\frac{1}{2} I+B\right)^{-1} A .
$$

В случае бесконечной гладкости поверхности $\Gamma$ и коэффициентов в $L$ операторы $A$ и $N$ - (сильно эллиптические) ПДО (псевдодифференциальные операторы) порядка -1 , а $H$ и $D-$ порядка 1.

3.2. Теперь приведем два варианта составления уравнений на $Г$ для смешанной задачи. В первом мы следуем работе [30]; ср. также [42], [43].

Этот вариант состоит в следующем. Продолжим $g$ до функции из $H^{1 / 2}(\Gamma)$, сохранив для продолжения обозначение $g$. Теперь для решения $u$ нашей задачи мы имеем $u^{+}=g+g_{0}$, где $g_{0} \in \widetilde{H}^{1 / 2}\left(\Gamma_{2}\right)$. Если мы найдем $g_{0}$, то сможем вычислить $u$ как решение задачи Дирихле. С другой стороны, $h=\left.\left(D\left(g+g_{0}\right)\right)\right|_{\Gamma_{2}}$. Введем оператор

$$
D_{\Gamma_{2}} \varphi=\left.(D \varphi)\right|_{\Gamma_{2}}
$$

из $\widetilde{H}^{1 / 2}\left(\Gamma_{2}\right)$ в $H^{-1 / 2}\left(\Gamma_{2}\right)$. Он получен из $D$ сужением области определения и ограничением получающихся функций на $\Gamma_{2}$. Получаем уравнение для $g_{0}$ :

$$
D_{\Gamma_{2}} g_{0}=h_{0},
$$

где $h_{0}=h-\left.(D g)\right|_{\Gamma_{2}}-$ известная функция.

Теорема 3.1. Onератор $D_{\Gamma_{2}}$ обратим.

Доказательство. Первое из неравенств (3.8) «наследуется» оператором $D_{\Gamma_{2}}$ :

$$
\|\varphi\|_{\widetilde{H}^{1 / 2}\left(\Gamma_{2}\right)}^{2} \leqslant C_{1} \operatorname{Re}\left(D_{\Gamma_{2}} \varphi, \varphi\right)_{\Gamma_{2}} .
$$

Здесь в форме справа функции находятся в дуальных пространствах $H^{-1 / 2}\left(\Gamma_{2}\right)$ и $\widetilde{H}^{1 / 2}\left(\Gamma_{2}\right)$. Достаточно воспользоваться леммой Лакса-Мильграма.

Итак, первый подход состоит по существу в решении уравнения (3.11) с последующим решением, скажем, уравнения $A \psi=g+g_{0}$, после чего $u$ определяется по формуле $u=\mathscr{A} \psi$. Как видно из (3.5), можно строить решение и в виде потенциала двойного слоя. 
Второй подход аналогичен первому, только вместо задачи Дирихле используется задача Неймана. Продолжим $h$ до элемента из $H^{-1 / 2}(\Gamma)$. После этого $T^{+} u=h+h_{0}$, где $h_{0} \in \widetilde{H}^{-1 / 2}\left(\Gamma_{1}\right)$. Введем оператор

$$
N_{\Gamma_{1}} \psi=\left.(N \psi)\right|_{\Gamma_{1}}
$$

из $\widetilde{H}^{-1 / 2}\left(\Gamma_{1}\right)$ в $H^{1 / 2}\left(\Gamma_{1}\right)$. Для $h_{0}$ получим уравнение

$$
N_{\Gamma_{1}} h_{0}=g_{1}
$$

где $g_{1}$ - известная функция $g-\left.(N h)\right|_{\Gamma_{1}}$.

Теорема 3.2. Onератор $N_{\Gamma_{1}}$ обратим.

Доказательство. Второе неравенство в (3.8) для $N$ наследуется оператором $N_{\Gamma_{1}}$ :

$$
\|\psi\|_{\widetilde{H}^{-1 / 2}\left(\Gamma_{1}\right)}^{2} \leqslant C_{2} \operatorname{Re}\left(N_{\Gamma_{1}} \psi, \psi\right)_{\Gamma_{1}}
$$

Функции справа принадлежат дуальным пространствам $H^{1 / 2}\left(\Gamma_{1}\right)$ и $\widetilde{H}^{-1 / 2}\left(\Gamma_{1}\right)$, и мы опять применяем лемму Лакса-Мильграма.

Второй вариант состоит, таким образом, в том, что мы решаем сначала уравнение (3.14), затем, скажем, уравнение $H \varphi=h+h_{0}$, после чего находим решение в виде $u=-\mathscr{B} \varphi$. Можно строить решение и в виде потенциала простого слоя, cM. (3.7).

Замечания. 1. Операторы $N_{\Gamma_{1}}$ и $D_{\Gamma_{2}}$ для частей замкнутой липшицевой поверхности являются аналогами операторов $N$ и $D$ для всей поверхности и наследуют свойства последних - неравенства типа Гординга и обратимость. Операторы $A_{S}$ и $H_{S}$ с близкими свойствами рассмотрены в [4]. В специальных случаях они встречались, в частности, в [42], [43], [30].

2. Названия «Neumann-to-Dirichlet operator» заслуживает не только оператор $N_{\Gamma_{1}}$, но и оператор $D_{\Gamma_{1}}^{-1}$, действующий из $H^{-1 / 2}\left(\Gamma_{1}\right)$ в $\widetilde{H}^{-1 / 2}\left(\Gamma_{1}\right)$. Это операторы с разными областями определения и разными областями значений. На $\Gamma_{2}$ тоже есть два оператора, $N_{\Gamma_{2}}$ и $D_{\Gamma_{2}}^{-1}$.

Приложения операторов $N, D, N_{\Gamma_{1}}, D_{\Gamma_{2}}$ чрезвычайно многообразны, см., например, [23], [48] и приведенную там литературу.

\section{§4. Регулярность решений}

4.1. Пространства $H_{p}^{s}$ бесселевых потенциалов и пространства $B_{p}^{s}$ Бесова. (См., например, [47], [21], [29], [2].) Условимся считать, что

$$
s \in \mathbb{R}, \quad 1<p<\infty, \quad p+p^{\prime}=p p^{\prime} .
$$

Пространство $H_{p}^{s}\left(\mathbb{R}^{n}\right)$ определяется формулой

$$
H_{p}^{s}\left(\mathbb{R}^{n}\right)=J^{-s} L_{p}\left(\mathbb{R}^{n}\right)
$$

где $J^{-s}$ - тот же оператор, что и в (1.6). При целом $s \geqslant 0$ это пространство Соболева $W_{p}^{s}\left(\mathbb{R}^{n}\right)$.

Пространства Слободецкого $W_{p}^{s}\left(\mathbb{R}^{n}\right)$ определяются при нецелых $s>0$. Если $0<s<1$, то

$$
\|u\|_{W_{p}^{s}\left(\mathbb{R}^{n}\right)}^{p}=\|u\|_{L_{p}\left(\mathbb{R}^{n}\right)}^{p}+\iint \frac{|u(y)-u(x)|^{p}}{|y-x|^{n+s p}} d x d y
$$


Пространство Бесова $B_{p}^{s}\left(\mathbb{R}^{n}\right)=B_{p, p}^{s}\left(\mathbb{R}^{n}\right)$ определяется формулой

$$
B_{p}^{s}\left(\mathbb{R}^{n}\right)=J^{\sigma-s} W_{p}^{\sigma}\left(\mathbb{R}^{n}\right), \quad 0<\sigma<1,
$$

при изменении $\sigma \in(0,1)$ норма заменяется эквивалентной нормой. При нецелом $s>0$ это пространства Слободецкого.

При $p=2$ пространства $H_{p}^{s}$ и $B_{p}^{s}$ совпадают с $H^{s}$.

Ниже в этом пункте букву $H$ можно заменить на $B$.

Пространства $H_{p}^{s}\left(\mathbb{R}^{n}\right)$ и $H_{p^{\prime}}^{-s}\left(\mathbb{R}^{n}\right)$ дуальны относительно продолжения стандартного скалярного произведения в $L_{2}\left(\mathbb{R}^{n}\right)$ на их прямое произведение.

Пространство $H_{p}^{s}(\Omega)$ состоит из сужений элементов из $H_{p}^{s}\left(\mathbb{R}^{n}\right)$ на $\Omega$ с нормой inf. Тот же самый, что и раньше, оператор $\mathscr{E}$ является ограниченным оператором продолжения элементов из $H_{p}^{s}(\Omega)$ до элементов из $H_{p}^{s}\left(\mathbb{R}^{n}\right)$ [37].

Пространство $\widetilde{H}_{p}^{s}(\Omega)$ определяется как подпространство в $H_{p}^{s}\left(\mathbb{R}^{n}\right)$ элементов с носителями в $\bar{\Omega}$. При $-1 / p^{\prime}<s<1+1 / p, s \neq 1 / p$ его можно отождествить с пополнением $\stackrel{\circ}{H}_{p}^{s}(\Omega)$ линеала $C_{0}^{\infty}(\Omega)$ в $H_{p}^{s}(\Omega)$.

Пространства $\widetilde{H}_{p^{\prime}}^{-s}(\Omega)$ и $H_{p}^{s}(\Omega)$ дуальны относительно формы (1.7). Пространства $H_{p}^{s}(\Omega)$ и $\widetilde{H}_{p}^{s}(\Omega)$ отождествляются при $-1 / p^{\prime}<s<1 / p$.

Пространства $B_{p}^{s}(\Gamma)$ вводятся для $|s| \leqslant 1$ при помощи разбиения единицы на Г и норм в $B_{p}^{s}\left(\mathbb{R}^{n-1}\right)$. Пространства $B_{p}^{s}(\Gamma)$ и $B_{p^{\prime}}^{-s}(\Gamma)$ дуальны относительно продолжения скалярного произведения в $L_{2}(\Gamma)$ на их прямое произведение.

Оператор перехода к следу действует ограниченным образом из $H_{p}^{s+1 / p}(\Omega)$ и из $B_{p}^{s+1 / p}(\Omega)$ в $B_{p}^{s}(\Gamma)$ при $0<s<1$. Эти два оператора имеют общий правый обратный $[22]$.

Пусть $\Gamma_{0}$ - область на $Г$. Пространство $B_{p}^{s}\left(\Gamma_{0}\right)$ определяется как состоящее из сужений на $\Gamma_{0}$ элементов из $B_{p}^{s}(\Gamma)$ с нормой inf. Имеется ограниченный оператор продолжения элементов из $B_{p}^{s}\left(\Gamma_{0}\right)$ до элементов из $B_{p}^{s}(\Gamma)$, не зависящий от $s, p$. Пространство $\widetilde{B}_{p}^{s}\left(\Gamma_{0}\right)$ определяется как подпространство в $B_{p}^{s}(\Gamma)$ элементов с носителями в $\bar{\Gamma}_{0}$. Пространства $\widetilde{B}_{p^{\prime}}^{-s}\left(\Gamma_{0}\right)$ и $B_{p}^{s}\left(\Gamma_{0}\right)$ дуальны относительно продолжения стандартного скалярного произведения в $L_{2}\left(\Gamma_{0}\right)$ на их прямое произведение. Пространства $B_{p}^{s}\left(\Gamma_{0}\right)$ и $\widetilde{B}_{p}^{s}\left(\Gamma_{0}\right)$ отождествляются при $-1 / p^{\prime}<s<1 / p$.

4.2. Решения смешанной задачи теперь ищутся в $H_{p}^{1 / 2+s+1 / p}(\Omega)$, где обязательно $|s|<1 / 2$ : в $(1.2)$

$$
g \in B_{p}^{1 / 2+s}\left(\Gamma_{1}\right), \quad h \in B_{p}^{-1 / 2+s}\left(\Gamma_{2}\right) .
$$

Вариационная (слабая) постановка смешанной задачи сохраняет вид (2.5). Пробные функции $v$ принадлежат подпространству $H_{p^{\prime}}^{1 / 2-s+1 / p^{\prime}}\left(\Omega, \Gamma_{1}\right)$ функций пространства $H_{p^{\prime}}^{1 / 2-s+1 / p^{\prime}}(\Omega)$ с нулевым следом на $\Gamma_{1}$. Сp. [27].

Правая часть $f$ системы принадлежит сопряженному пространству

$$
\widetilde{H}_{p}^{-1 / 2+s-1 / p^{\prime}}\left(\Omega, \Gamma_{1}\right):=\left[H_{p^{\prime}}^{1 / 2-s+1 / p^{\prime}}\left(\Omega, \Gamma_{1}\right)\right]^{*}
$$

относительно продолжения формы (1.7).

Здесь всюду пространства $H$ можно заменить пространствами $B$. 
Допустимые точки $(s, t), t=1 / p$, образуют квадрат

$$
Q=\{(s, t):|s|<1 / 2,0<t<1\} .
$$

Частные случаи смешанной задачи - задачи Дирихле и Неймана. Про них мы знаем [3], что они однозначно разрешимы при $|s|<\varepsilon,|t-1 / 2|<\delta$ с достаточно малыми $\varepsilon$ и $\delta$. Мы хотим получить аналогичный результат для смешанной задачи. Мы сделаем это, как в [4], рассматривая операторы типа потенциала в шкалах пространств на границе. Ср. [17], [27], [11].

Прежде всего заметим, что операторы

$$
\begin{array}{cc}
A, N: B_{p}^{-1 / 2+s}(\Gamma) \rightarrow B_{p}^{1 / 2+s}(\Gamma), & H, D: B_{p}^{1 / 2+s}(\Gamma) \rightarrow B_{p}^{-1 / 2+s}(\Gamma), \\
\frac{1}{2} I \pm B: B_{p}^{1 / 2+s}(\Gamma) \rightarrow B_{p}^{1 / 2+s}(\Gamma), & \frac{1}{2} I \pm \widehat{B}: B_{p}^{-1 / 2+s}(\Gamma) \rightarrow B_{p}^{-1 / 2+s}(\Gamma)
\end{array}
$$

ограничены и обратимы при тех же $(s, t)[3]$. При этом $N=D^{-1}$ и сохраняются соотношения (3.9).

Оператор $N_{\Gamma_{1}}$ действует ограниченным образом из $\widetilde{B}_{p}^{-1 / 2+s}\left(\Gamma_{1}\right)$ в $B_{p}^{1 / 2+s}\left(\Gamma_{1}\right)$ (при тех же $(s, t))$. Каждое из этих двух семейств пространств образует интерполяционную шкалу относительно комплексного метода интерполяции по каждому из индексов. Мы объяснили это в [4] при рассмотрении операторов $A_{S}$ и $H_{S}$. В точке $(s, t)=(0,1 / 2)$ наш оператор обратим. Значит, применима теорема Шнейберга [40], и получается

Tеорема 4.1. Onepamop $N_{\Gamma_{1}}: \widetilde{B}_{p}^{-1 / 2+s}\left(\Gamma_{1}\right) \rightarrow B_{p}^{1 / 2+s}\left(\Gamma_{1}\right)$ остается обратимым при $|s|<\varepsilon,|t-1 / 2|<\delta$ с достаточно малыми $\varepsilon$ и $\delta$.

При этих $(s, t)$ мы сейчас установим однозначную разрешимость смешанной задачи. Сначала проверим единственность. Пусть $g=0$ и $h=0$. Тогда $T^{+} u=h_{0} \in \widetilde{B}_{p}^{-1 / 2+s}\left(\Gamma_{1}\right)$. Значит, $N h_{0}=u^{+}$на $\Gamma$ и $N_{\Gamma_{1}} h_{0}=0$. Но оператор $N_{\Gamma_{1}}$ обратим, поэтому $h_{0}=0$ и $T^{+} u=0$ на $Г$. Остается воспользоваться единственностью для задачи Неймана. Мы использовали второй вариант подхода $\mathrm{K}$ смешанной задаче из п. 3.2. С этим вариантом очевидным образом получается и существование. Это дает основной результат в настоящем пункте.

Теорема 4.2. Смешанная задача (1.1)-(1.2) с $f=0, g \in B_{p}^{1 / 2+s}\left(\Gamma_{1}\right), h \in$ $B_{p}^{-1 / 2+s}\left(\Gamma_{2}\right)$ остается однозначно разрешимой при $|s|<\varepsilon,|t-1 / 2|<\delta$ c достаточно малыми $\varepsilon$ и .

В этих рамках, чем «лучше» правые части, тем «лучше» решение. Это и есть результат о регулярности. Он автоматически следует из теоремы 4.2.

Замечания. 1. Вместо $N_{\Gamma_{1}}$ можно использовать оператор $D_{\Gamma_{2}}$, действующий из $\widetilde{B}_{p}^{1 / 2+s}\left(\Gamma_{2}\right)$ в $B_{p}^{-1 / 2+s}\left(\Gamma_{2}\right)$, для него тоже получается ограниченность $u$ обратимость при $|s|<\varepsilon,|t-1 / 2|<\delta$ с достаточно малыми $\varepsilon$ и $\delta$. Затем используется первый вариант подхода к смешанной задаче из п. 3.2.

2. Как мы видим, в этих более общих пространствах сохраняются оба варианта сведе́ния смешанной задачи $к$ эквивалентным уравнениям на границе и решения этих уравнений при помощи операторов типа потенциала.

3. Теорема 4.2 обобщается на случай ненулевой правой части $f$ в уравнении $L u=f$. Действительно, пусть $f \in \widetilde{H}_{p}^{-1 / 2+s-1 / p^{\prime}}\left(\Omega, \Gamma_{1}\right)$. Тогда мы можем считать $f$ элементом пространства $\widetilde{H}_{p}^{-1 / 2+s-1 / p^{\prime}}(\Omega)$. (Букву $H$ можно заменить буквой $B$.$) Пусть u_{0}$ - решение задачи Неймана для уравнения $L u_{0}=f \mathrm{c}$ 
нулевой конормальной производной. Вычитая $u_{0}$ из $u$, приходим к задаче, к которой применима теорема 4.2.

\section{§5. Спектральные задачи}

5.1. Задача со спектральным параметром в системе. Рассмотрим задачу

$$
L u=\lambda u \quad \text { в } \Omega, \quad u^{+}=0 \quad \text { на } \Gamma_{1}, \quad T^{+} u=0 \quad \text { на } \Gamma_{2} .
$$

Пусть сначала оператор $L$ формально самосопряженный: $L=\widetilde{L}$. Считая уравнение $L u=f$ однозначно разрешимым в $H^{1}\left(\Omega, \Gamma_{1}\right)$ при $f \in \widetilde{H}^{-1}\left(\Omega, \Gamma_{1}\right)$, введем в последнем пространстве скалярное произведение (ср. [34])

$$
\left\langle f_{1}, f_{2}\right\rangle_{\Omega}:=\left(L^{-1} f_{1}, f_{2}\right)_{\Omega} .
$$

Относительно него неограниченный оператор $L$ в этом пространстве (с областью определения $\left.H^{1}\left(\Omega, \Gamma_{1}\right)\right)$ остается самосопряженным. Собственные значения положительны. Как и в других задачах, рассмотренных раньше в [3] и [5], собственные значения и собственные функции совпадают с собственными значениями и собственными функциями аналогичного оператора в $L_{2}(\Omega)$. А так как пространство $H^{1}\left(\Omega, \Gamma_{1}\right)$ содержит $\widetilde{H}^{1}(\Omega)=\stackrel{\circ}{H}^{1}(\Omega)$ и содержится в $H^{1}(\Omega)$, то действует результат Метивье [25], согласно которому для считающей функции $N_{L}(\lambda)$ - числа собственных значений с учетом кратностей, меньших $\lambda$, справедлива асимптотика

$$
N_{L}(\lambda)=c_{L} \lambda^{n / 2}+O\left(\lambda^{(n-1 / 2) / 2}\right),
$$

где коэффициент $c_{L}$ - тот же, что и в задачах Дирихле и Неймана (см. [5]).

Далее, справедливы такие же утверждения о собственных функциях, как в случаях этих задач [5]. Из собственных функций составляется ортонормированный базис в $\widetilde{H}^{-1}\left(\Omega, \Gamma_{1}\right)$. Они принадлежат пространству $H^{1}\left(\Omega, \Gamma_{1}\right)$ и там образуют ортонормированный базис относительно скалярного произведения $(L u, v)_{\Omega}$, кстати, равного $\Phi_{\Omega}(u, v)$. Этот результат распространяется на промежуточные пространства. Более того, можно удлинить шкалу этих пространств влево и вправо на $\varepsilon$. Если же выйти в пространства, отвечающие значениям $t$ с $|t-1 / 2|<\delta, t \neq 1 / 2$, то там сохраняется полнота собственных функций и суммируемость рядов Фурье по ним методом Абеля-Лидского.

Если только главная часть оператора $L$ формально самосопряженная, то собственные значения лежат в сколь угодно узком угле с биссектрисой $\mathbb{R}_{+}$с некоторого номера и имеют асимптотику с тем же главным членом. Сохраняются утверждения о гладкости корневых функций, об их полноте и о суммируемости.

Если совсем отказаться от предположений о самосопряженности, то сохраняется оценка собственных значений $\left|\lambda_{j}\left(L^{-1}\right)\right| \leqslant C j^{-2 / n}$. Наибольшая общность, в которой мы можем получить утверждения о полноте и о суммируемости, это случай, когда все значения формы $\Phi_{\Omega}(u, u)$ лежат в угле раствора меньше $2 \pi / n$ с биссектрисой $\mathbb{R}_{+}$. Сp. [5].

5.2. Задачи Пуанкаре-Стеклова со спектральным параметром на части границы. Рассмотрим две задачи.

$$
\text { I. } L u=0 \quad \text { в } \Omega, \quad T^{+} u=0 \quad \text { на } \Gamma_{2}, \quad \lambda T^{+} u=u^{+} \text {на } \Gamma_{1} .
$$


Здесь $T^{+} u \in \widetilde{H}^{-1 / 2}\left(\Gamma_{1}\right)$ и (см. п. 3.2) $N_{\Gamma_{1}} T^{+} u=u^{+} \in H^{1 / 2}\left(\Gamma_{1}\right)$. Поэтому для собственных функций спектральной задачи I получается уравнение $N_{\Gamma_{1}} \psi=$ $\lambda \psi$, где $\psi=T^{+} u$, и оно эквивалентно этой задаче при $L=\widetilde{L}$.

$$
\text { II. } L u=0 \quad \text { в } \Omega, \quad u^{+}=0 \text { на } \Gamma_{2}, \quad \lambda T^{+} u=u^{+} \quad \text { на } \Gamma_{1} \text {. }
$$

Здесь $u^{+} \in \widetilde{H}^{1 / 2}\left(\Gamma_{1}\right)$ и (см. п. 3.2) $D_{\Gamma_{1}} u^{+}=T^{+} u \in H^{-1 / 2}\left(\Gamma_{1}\right)$. Поэтому для собственных функций спектральной задачи II получается уравнение $D_{\Gamma_{1}}^{-1} \psi=$ $\lambda \psi$, где снова $\psi=T^{+} u$, и оно эквивалентно этой задаче при $L=\widetilde{L}$.

Во второй задаче мы поменяли местами $\Gamma_{1}$ и $\Gamma_{2}$ по сравнению с п. 3.2 , чтобы ниже сопоставить соответствующие операторы.

Спектральные свойства операторов $N_{\Gamma_{1}}$ и $D_{\Gamma_{1}}$ аналогичны спектральным свойствам соответственно операторов $A_{S}$ и $H_{S}$ (при $\Gamma_{1}=S$ ), описанным в [4]. Скалярное произведение $\left\langle\psi_{1}, \psi_{2}\right\rangle_{\Gamma_{1}}$ при $L=\widetilde{L}$ определяем в первом случае в $\widetilde{H}^{-1 / 2}\left(\Gamma_{1}\right)$ как $\left(N_{\Gamma_{1}} \psi_{1}, \psi_{2}\right)_{\Gamma_{1}}$ и во втором случае в $H^{-1 / 2}\left(\Gamma_{1}\right)$ как $\left(D_{\Gamma_{1}}^{-1} \psi_{1}, \psi_{2}\right)_{\Gamma_{1}}$.

В частности, мы имеем в виду свойства гладкости собственных и корневых функций, результаты об ортонормированном базисе из собственных функций при $p=2$, если $L=\widetilde{L}$, о полноте и суммируемости рядов по корневым функциям методом Абеля-Лидского в остальных случаях. Если оператор $L$ имеет формально самосопряженную главную часть, то собственные значения лежат в сколь угодно узком угле с биссектрисой $\mathbb{R}_{+}$с некоторого номера. Наибольшая общность, при которой получаются утверждения о полноте и суммируемости, - это случай, когда все значения квадратичных форм $\Phi_{\Omega^{ \pm}}(u, u)$ лежат в угле раствора меньше $\pi /(n-1)$ с биссектрисой $\mathbb{R}_{+}$.

Дополнительные результаты о полноте получаются как следствия (плотных) вложений рассматриваемых пространств.

\section{§6. Спектральные асимптотики}

Здесь мы хотим получить асимптотические формулы для собственных значений операторов $N_{\Gamma_{1}}$ и $D_{\Gamma_{1}}^{-1}$. Но до этого рассмотрим операторы на Г. Сформулируем сначала следующие предположения.

$1^{\circ} . L=\widetilde{L}$.

$2^{\circ}$. Поверхность Г является почти гладкой (см. п. 1.1).

$3^{\circ} . L-$ скалярный оператор или матричный оператор, старшая часть которого совпадает с оператором Ламе.

Как показано в [5], при этих условиях можно вывести асимптотическую формулу для собственных значений $\lambda_{j}(N)$ оператора $N$

$$
\lambda_{j}(N) \sim C_{N} j^{-1 /(n-1)}
$$

(в том смысле, что разность левой и правой частей есть $\left.o\left(j^{-1 /(n-1)}\right)\right)$ из результатов работы [6] в сочетании с приведенной выше формулой (3.9) и очень глубокими известными результатами об обратимости операторов $\frac{1}{2} I \pm B$ и $\frac{1}{2} I \pm \widehat{B}$ в $L_{2}(\Gamma)$ [28], [14]. Собственные значения нумеруются в порядке невозрастания с учетом кратностей.

Мы освободимся здесь от ограничения $3^{\circ}$ при помощи вариационного подхода к асимптотикам. При этом будем рассматривать оператор $b N$, где функцию 
$b$ будем для начала считать мультипликатором в $H^{ \pm 1 / 2}(\Gamma)$ и для простоты неотрицательной функцией. Липшицеву поверхность Г будем считать почти гладкой в окрестности ее носителя. Результат сформулирован ниже в теореме 6.5. Cp. с [1] и в особенности с работой [45], где рассматривались кусочно-гладкие поверхности.

Пусть $T$ - компактный самосопряженный оператор в гильбертовом пространстве $H$ со скалярным произведением $(\cdot, \cdot)$, для простоты неотрицательный - с неотрицательными собственными значениями. Считающая функция $N(\lambda)=N(\lambda, T)$ для его положительных собственных значений $\lambda_{j}(T)(j=$ $1,2, \ldots)$ - это число собственных значений с учетом кратностей, бо́льших $\lambda$ $(\lambda>0)$. Асимптотика $N(\lambda) \sim \beta \lambda^{-\alpha}$ при $\lambda \rightarrow 0(\alpha>0, \beta>0)$ эквивалентна асимптотике $\lambda_{j} \sim \beta^{\prime} j^{-1 / \alpha}$ при $j \rightarrow \infty, \beta^{\prime}=\beta^{1 / \alpha}$.

Вариационное отношение для $T$ имеет вид $R(x)=(T x, x) /(x, x)$. Собственные значения являются «последовательными максимумами» этого отношения:

$$
\lambda_{j+1}(T)=\min _{\operatorname{codim} X \leqslant j} \max _{0 \neq x \in X} R(x),
$$

где $X$ - подпространства в $H$.

Нам понадобятся известные леммы, мы приведем их в упрощенном виде (ср. $[8$, добавление 1] или $[45$, п. 1]). Первая из них позволяет сравнивать считающие функции операторов, действующих в разных пространствах.

Лемма 6.1. Пусть $H_{1}$ и $H_{2}$ - гильбертовы пространства со скалярными произведениями $(\cdot, \cdot)_{1},(\cdot, \cdot)_{2}, T_{1}$ и $T_{2}-$ компактные неотрицательные операторы в этих пространствах, $S$ - ограниченный оператор из $\mathrm{H}_{1}$ в $\mathrm{H}_{2}$, $\left(T_{1} x, x\right)_{1}=0$ при $S x=0$ u

$$
\left(T_{1} x, x\right)_{1} /(x, x)_{1} \leqslant\left(T_{2} S x, S x\right)_{2} /(S x, S x)_{2} \quad\left(x \in H_{1}, S x \neq 0\right) .
$$

Тогда $N\left(\lambda, T_{1}\right) \leqslant N\left(\lambda, T_{2}\right)$. В частности, это верно, если $H_{1} \subset H_{2},(\cdot, \cdot)_{1}=$ $(\cdot, \cdot)_{2}$ на $H_{1}$ и $S$ - оператор вложения; знаменатели в (6.3) в этом случае не нужны.

Лемма 6.2. Пусть $H$ - ортогональная сумма $H_{1} \oplus H_{2}$, подпространства $H_{1}$ и $H_{2}$ инвариантны относительно неотрицательного оператора $T$ в $H u$ $T_{j}$ - сужения этого оператора на $H_{j}$. Тогда $N(\lambda, T)=N\left(\lambda, T_{1}\right)+N\left(\lambda, T_{2}\right)$.

Лемма 6.3 (М. Ш. Бирман-М. З. Соломяк). Пусть компактный неотрии,ательный оператор $T$ в гилъбертовом пространстве $H$ при любом $\varepsilon>0$ допускает представление $T=T_{\varepsilon}^{\prime}+T_{\varepsilon}^{\prime \prime}$, где $T_{\varepsilon}^{\prime} u T_{\varepsilon}^{\prime \prime}-$ компактные операторы, $T_{\varepsilon}^{\prime}$ неотрицателен, $\lambda_{j}\left(T_{\varepsilon}^{\prime}\right) \sim C\left(T_{\varepsilon}^{\prime}\right) j^{-\sigma}$ при некотором $\sigma>0 u \lim \sup \left|\lambda_{j}\left(T_{\varepsilon}^{\prime \prime}\right)\right| j^{\sigma}$ $\leqslant \varepsilon$. Тогда при $\varepsilon \rightarrow 0$ величина $C\left(T_{\varepsilon}^{\prime}\right)$ имеет конечный предел $C(T)$ и $\lambda_{j}(T) \sim$ $C(T) j^{-\sigma}$.

6.1. Оператор $b N$. Коэффициенты в $L$ будем сначала считать бесконечно гладкими. Введем в $H^{-1 / 2}(\Gamma)$ скалярное произведение

$$
\left\langle\psi_{1}, \psi_{2}\right\rangle_{\Gamma}=\left(N \psi_{1}, \psi_{2}\right)_{\Gamma} .
$$

Оператор $b N$ остается компактным и самосопряженным. Рассмотрим вариационное отношение

$$
R_{b}(\psi)=\langle b N \psi, \psi\rangle_{\Gamma} /\langle\psi, \psi\rangle_{\Gamma}=(b N \psi, N \psi)_{\Gamma} /(N \psi, \psi)_{\Gamma} \quad\left(\psi \in H^{-1 / 2}(\Gamma)\right) .
$$


Считая, что $\psi=T^{+} u$ для решения $u \in H^{1}(\Omega)$ системы $L u=0$, имеем $N \psi=$ $u^{+}$. Поэтому числитель равен $\left(b u^{+}, u^{+}\right)_{\Gamma}$, а знаменатель равен $\left(T^{+} u, u^{+}\right)_{\Gamma}$, т. е. $\Phi_{\Omega}(u, u)$ в силу формулы Грина. Отношение (6.5) переписывается в виде

$$
Q_{b}(u)=\left(b u^{+}, u^{+}\right)_{\Gamma} / \Phi_{\Omega}(u, u) .
$$

Теперь числитель - форма некоторого компактного оператора в подпространстве пространства $H^{1}(\Omega)$, состоящем из решений $u$ системы $L u=0$. За скалярное произведение в $H^{1}(\Omega)$ принимаем $\Phi_{\Omega}(u, v)$. Тогда ортогональное дополнение к подпространству решений составляют функции с $u^{+}=0$, это уже отмечалось в п. 2.1. Поэтому (см. лемму 6.2) будем рассматривать отношение (6.6) на всех $u \in H^{1}(\Omega)$. (На самом деле переход от (6.5) к (6.6) не обязателен.)

Теперь воспользуемся тем, что для липшицевой области $\Omega$ можно построить области $\widetilde{\Omega}$ и $\widehat{\Omega}$ с бесконечно гладкими границами $\widetilde{\Gamma}$ и $\widehat{\Gamma}$, такие, что $\widetilde{\Omega} \subset \Omega \subset \widehat{\Omega}$ и эти границы сколь угодно близки к Г. Более точно, между этими гладкими границами и Г имеется взаимно однозначное соответствие и расстояние между соответствующими точками становится равномерно сколь угодно малым. См., например, [49].

В нашей ситуации, зафиксировав окрестность $U$ сингулярного множества $\Gamma_{\text {sing }}$ со сколь угодно малой мерой, мы можем принять, что $\widetilde{\Gamma}$ и $\widehat{\Gamma}$ совпадают $c \Gamma$ вне $U$. Пусть $\theta_{U}$ - гладкое приближение к характеристической функции дополнения к $U$ с носителем в этом дополнении. Тогда для соответствующих отношений $\widetilde{Q}_{b \theta_{U}}(u)$ и $\widehat{Q}_{b \theta_{U}}(u)$ мы имеем

$$
\widehat{Q}_{b \theta_{U}}(u) \leqslant Q_{b \theta_{U}}(u) \leqslant \widetilde{Q}_{b \theta_{U}}(u)
$$

на функциях из $H^{1}(\widehat{\Omega})$ и их сужениях на $\Omega$ и $\widetilde{\Omega}$. Левое и правое отношения отвечают гладким задачам, и соответствующие операторы $b \theta_{U} \widehat{N}$ и $b \theta_{U} \widetilde{N}$ имеют известную и одинаковую асимптотику собственных значений (мы имеем в виду ее старший член), см. [9]. Отсюда аналогичный результат получается для $b \theta_{U} N$. Это вытекает из леммы 6.1.

Теперь рассмотрим отношение $Q_{c}(u)$, где $c=b\left(1-\theta_{U}\right)$. В силу ограниченности оператора перехода к следу

$$
Q_{c}(u) \leqslant C \int_{\Gamma}\left|c\left\|\left.u\right|^{2} d S /\right\| u \|_{H^{1 / 2}(\Gamma)}^{2},\right.
$$

и справа $Г$ можно заменить на $S=\operatorname{supp} c$. После этого отношение справа отвечает компактному неотрицательному оператору в $H^{1 / 2}(S)$.

Лемма 6.4. Пусть $S$ - область на ограниченной липшицевой поверхности Г размерности $n-1$ с липшищевой гранищей и $c(x)$ - неотрищательная функиия из $L_{r}(S)$ на этой поверхности. Тогда для считающей функции $N(\lambda, T)$ компактного оператора $T$ в $H^{s}(S), s>0$, с вариационным отношением

$$
\int_{S} c(x)|u(x)|^{2} d S /\|u\|_{H^{s}(S)}^{2}
$$

справедливо неравенство

$$
N(\lambda, T) \leqslant C_{1}\|c\|_{L_{r}(S)}^{\tau} \lambda^{-\tau}
$$

при $r>1$, если $n-1=2 s$, u $r=(n-1) /(2 s)$, если $n-1>2 s$, c $\tau=(n-1) /(2 s)$. 
Доказательство. В случае, когда $S$ - область в $\mathbb{R}^{n-1}$, это лемма 1.7 в [45]. Как там указано, это утверждение получено в [8] (теорема 4.1) для целых $s$, но доказательство сохраняется при нецелых $s$. Лемма установлена для скалярных функций, но легко переносится на вектор-функции.

Чтобы распространить ее на случай области на липшицевой поверхности, можно принять, что эта область мала и допускает представление $x_{n}=\phi\left(x^{\prime}\right)$ с липшицевой функцией $\phi\left(x^{\prime}\right)$ в проекции этой области на $x^{\prime}$-плоскость. Остается учесть, что $d S=\left(1+\left|\nabla \phi\left(x^{\prime}\right)\right|^{2}\right)^{1 / 2} d x^{\prime}$ и градиент здесь ограничен.

В нашей ситуации $s=1 / 2$, и лемма приводит к оценке

$$
\left|\lambda_{j}(T)\right| \leqslant C_{2}\|c\|_{L_{r}(S)} \lambda^{-(n-1)},
$$

решающей дело, поскольку $L_{r}$-норма функции $c=b\left(1-\theta_{U}\right)$ стремится к нулю, когда окрестность $U$ стягивается к $\Gamma_{\text {sing }}$, так как при наших предположениях о $b$ это ограниченная функция. Здесь следует воспользоваться леммой 6.3 .

Предположение о гладкости коэффициентов в $L$ снимается при помощи аппроксимации исходных коэффициентов гладкими (ср. [45, п. 5]). Получается

Теорема 6.5. Пусть $L=\widetilde{L}$, неотрицательная функция $b-м$ мльтипликатор в $H^{ \pm 1 / 2}(\Gamma)$ и поверхность Г является почти гладкой в окрестности ее носителя. Тогда для собственных значений оператора $b N$ справедлива асимптотика вида (6.1).

Коэффициент $C_{b N}$ вычисляется по формуле

$$
C_{b N}^{n-1}=(2 \pi)^{-(n-1)} \iint_{T^{*} \Gamma} b\left(x^{\prime}\right) n_{\alpha}\left(x^{\prime}, \xi^{\prime}\right) d x^{\prime} d \xi^{\prime}
$$

в случае гладких коэффициентов в $L$; здесь из $\Gamma$ выбрасывается сингулярное множество. Через $\alpha\left(x^{\prime}, \xi^{\prime}\right)$ обозначен главный символ оператора $N$ и через $n_{\alpha}\left(x^{\prime}, \xi^{\prime}\right)$ число его собственных значений, больших 1 . Если коэффициенты в $L$ негладкие, то при их аппроксимации гладкими операторы $b N$ для этих гладких задач сходятся по операторной норме к интересующему нас оператору. Нужный коэффициент получается предельным переходом, предел существует в силу той же леммы 6.3. Он вычисляется через главный символ оператора $L$.

Из-за недостатка места мы не приводим обобщение на случай функции $b$ со значениями разных знаков. Однако отметим следующее обобщение.

Теорема 6.6. Утверждение теоремы 6.5 остается в силе в случае функиии $b$, принадлежащей $L_{r}(\Gamma)$, где $r=n-1$ при $n>2 u r>1$ при $n=2$, если определить $b N$ как оператор с вариачионным отношением (6.5) или (6.6).

При использовании, например, отношения (6.6) имеется в виду оператор в $H^{1}(\Omega)$, имеющий форму $\left(b u^{+}, v^{+}\right)_{\Gamma}$. Эта форма определена в силу теорем вложения и неравенства Гёльдера. В этом случае $b$ уже, вообще говоря, не является мультипликатором в $H^{ \pm 1 / 2}(\Gamma)$.

6.2. Оператор $N_{\Gamma_{1}}$. В этом случае в качестве $b$ хотелось бы взять характеристическую функцию $\theta$ области $\Gamma_{1}$. Но это не мультипликатор в $H^{1 / 2}(\Gamma)$. Однако эта функция является мультипликатором в $\widetilde{H}^{-1 / 2}\left(\Gamma_{1}\right)$; более того, умножение на нее не меняет элементы этого пространства. Это позволяет переписать уравнение $N_{\Gamma_{1}} \psi=\lambda \psi$ в виде 
по крайней мере, считая $u^{+}$функцией из $H^{1 / 2-\varepsilon}\left(\Gamma_{1}\right)$ со сколь угодно малым $\varepsilon>0$. Соответствующее вариационное отношение имеет вид

$$
\left(\theta u^{+}, u^{+}\right)_{\Gamma} / \Phi_{\Omega}(u, u)
$$

Здесь $u$ принадлежит подпространству решений системы $L u=0$ в $H^{1}(\Omega)$ с $T^{+} u=0$ вне $\Gamma_{1}$, и теперь можно считать, что $u^{+} \in H^{1 / 2}\left(\Gamma_{1}\right)$. В силу формулы Грина

$$
\Phi_{\Omega}(u, v)=\left(T^{+} u, v^{+}\right)_{\Gamma_{1}}
$$

ортогональное дополнение к указанному подпространству состоит из функций $u$ с $u^{+}=0$ на $\Gamma_{1}$. На них отношение (6.14) равно нулю. Поэтому его можно рассматривать на всех $u \in H^{1}(\Omega)$ с $L u=0$ и, далее, на всем $H^{1}(\Omega)$. Теперь применима теорема 6.6 , и мы приходим к желаемому результату:

Теорема 6.7. Пусть $L=\widetilde{L}$ и поверхность Г почти гладкая в окрестности замыкания области $\Gamma_{1}$. Тогда имеет место асимптотика

$$
\lambda_{j}\left(N_{\Gamma_{1}}\right) \sim C_{N_{\Gamma_{1}}} j^{-1 /(n-1)} .
$$

Здесъ $C_{N_{\Gamma_{1}}}$ определяется формулой вида (6.12) с b=1 и $\Gamma_{1}$ вместо Г.

6.3. Оператор $D_{\Gamma_{1}}^{-1}$. Основной результат в этом пункте - теорема $6.8-$ получен автором совместно с Т. А. Суслиной.

Оператор $D_{\Gamma_{1}}^{-1}$ - это оператор, отвечающий задаче Дирихле с $u^{+}=0$ вне $\Gamma_{1}$. Для него вариационное отношение имеет вид

$$
\left(u^{+}, u^{+}\right)_{\Gamma_{1}} /\left(D u^{+}, u^{+}\right)_{\Gamma_{1}} \quad\left(L u=0 \text { в } \Omega, u^{+} \in \widetilde{H}^{1 / 2}\left(\Gamma_{1}\right)\right) .
$$

Асимптотика для гладких задач такого вида исследована в [44]. Ключевой момент в нашем случае состоит в проверке того, что отношение (6.17), если Г и

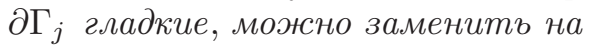

$$
\left(\theta u^{+}, u^{+}\right)_{\Gamma} /\left(D u^{+}, u^{+}\right)_{\Gamma} \quad\left(L u=0 \quad \text { в } \Omega, u^{+} \in H^{1 / 2}(\Gamma)\right)
$$

в смысле совпадения асимптотик. Относительно просто это можно сделать в нашем случае следующим образом. Мы дважды воспользуемся леммой 6.1.

Пусть функции $\alpha$ и $\beta$ принадлежат $C^{\infty}(\Gamma)$, их значения заключены между 0 и $1, \operatorname{supp} \alpha \subset \Gamma_{1}$ и $\alpha^{2}+\beta^{2}=1 ; \alpha$ аппроксимирует функцию $\theta$. Мы имеем, полагая $\varphi=u^{+}$,

$$
(\alpha \varphi, \alpha \varphi)_{\Gamma} /\left[(D \alpha \varphi, \alpha \varphi)_{\Gamma}+(D \beta \varphi, \beta \varphi)_{\Gamma}\right] \leqslant(\alpha \varphi, \alpha \varphi)_{\Gamma} /(D \alpha \varphi, \alpha \varphi)_{\Gamma} .
$$

Здесь $\varphi-$ функция из $H^{1 / 2}(\Gamma)$. В качестве $S$ берем отображение $\varphi \mapsto \alpha \varphi$ этого пространства в $\widetilde{H}^{1 / 2}\left(\Gamma_{1}\right)$. Добавочно заметим, что формы в знаменателях в (6.18) и слева в (6.19) совпадают с точностью до прибавления формы ПДО нулевого порядка ввиду совпадения главных символов. Известно, что такое прибавление не отражается на асимптотике (см. [8] или [45, п. 1]).

Теперь пусть $\alpha_{1}$ - неотрицательная функция из $C^{\infty}(\Gamma)$, равная 1 в окрестности замыкания области $\Gamma_{1}$, тоже аппроксимирующая $\theta$. Имеем

$$
(\varphi, \varphi)_{\Gamma} /(D \varphi, \varphi)_{\Gamma}=\left(\alpha_{1} \varphi, \alpha_{1} \varphi\right)_{\Gamma} /(D \varphi, \varphi)_{\Gamma}
$$

Здесь $\varphi-$ функция из $\widetilde{H}^{1 / 2}\left(\Gamma_{1}\right)$ и $S$ - вложение этого пространства в $H^{1 / 2}(\Gamma)$. 
Лемма 6.1 показывает, что считающая функция для оператора с отношением (6.17) заключена между считающими функциями для операторов с отношениями слева в $(6.19)$ и $\left(\alpha_{1} \varphi, \alpha_{1} \varphi\right)_{\Gamma} /(D \varphi, \varphi)_{\Gamma}, \varphi \in H^{1 / 2}(\Gamma)$. Асимптотика операторов с такими вариационными отношениями известна из [9]. Теперь предельный переход с использованием леммы 6.3 доказывает выделенное выше утверждение. После этого фактически вариационное отношение, а значит, и асимптотика такие же, как в п. 6.2.

На случай почти гладкой поверхности результат распространяется при помощи соображений, аналогичных использованным в п. 6.1. Получается

Теорема 6.8. Если $L=\widetilde{L}$ и поверхность Г почти гладкая в окрестности замыкания области $\Gamma_{1}$, то для собственных значений оператора $D_{\Gamma_{1}}^{-1}$ имеет место асимптотика вида (6.1) с тем же коэффициентом, что и для $N_{\Gamma_{1}}$.

Пример (ср. [23, с. 50]). Рассмотрим уравнение Лапласа в квадрате $\{(x, y)$ : $0<x<\pi, 0<y<\pi\}$. Пусть $\Gamma_{1}-$ его левая сторона, а $\Gamma_{2}$ состоит из трех других сторон. Задача I имеет решения $\operatorname{ch} k(\pi-x) \cos k y(k=0,1, \ldots)$. Задача II - решения $\operatorname{sh} k(\pi-x) \sin k y(k=1,2, \ldots)$. Собственные функции: $\cos k y$ в задаче I и $\sin k y$ в задаче II. Собственные значения: соответственно $\operatorname{cth} k \pi / k$ и th $k \pi / k$. Они разные в двух задачах, но асимптотика одинакова.

6.4. Замечания к работам [4] и [5]. Вариационное отношение для оператора $H^{-1}$ имеет вид (ср. [5, предложение 9.1])

$$
(\varphi, \varphi)_{\Gamma} /(H \varphi, \varphi)_{\Gamma} \quad\left(\varphi=[u] \in H^{1 / 2}(\Gamma), u=\mathscr{B} \varphi\right) .
$$

Здесь $[T u]=0$, и решения системы $L u=0$ в $\Omega^{ \pm}$определяются скачком $[u]$. Соображения из п. 6.1 в случае почти гладкой $Г$ применимы к (6.21) непосредственно. По сравнению с [5] удается снять условие $3^{\circ}$. Далее, для оператора $H_{S}^{-1}$ можно получить аналог теоремы 6.8 по существу как в предъдущем пункте. Подход в [4] к $H_{S}^{-1}$ нуждается в некоторой ревизии, возможной при помощи вариационных соображений, но в этом уже нет нужды.

$\mathrm{K}$ операторам $A$ и $A_{S}$ тоже возможен вариационный подход, но он не дает ничего нового по сравнению с [36] и [4]. Ограничимся замечанием, что вариационному отношению для $A$ можно придать вид $\left(u^{ \pm}, u^{ \pm}\right)_{\Gamma} / \Phi_{\mathbb{T}}(u, u)$.

\section{§7. Некоторые обобщения}

7.1. Более общие задачи Пуанкаре-Стеклова. Мы хотим теперь рассмотреть случай, когда граница $\Gamma$ разбита на три области $\Gamma_{1}, \Gamma_{2}$ и $\Gamma_{3}$ : в первой ставится однородное условие Дирихле, во второй - однородное условие Неймана и в третьей - спектральное условие Пуанкаре-Стеклова. Будем считать, что имеются две замкнутые липшицевы поверхности размерности $n-2$ без самопересечений и без общих точек, отделяющие эти области одну от другой. Cp. [34].

Рассмотрим для определенности случай, когда область $\Gamma_{3}$ разделяет области $\Gamma_{1}$ и $\Gamma_{2}$. Введем следующие обозначения. Через $\Gamma_{2,3}$ обозначим дополнение $\mathrm{k}$ $\bar{\Gamma}_{1}$ и через $\Gamma_{1,3}-$ дополнение к $\bar{\Gamma}_{2}$. Пусть $\widehat{H}^{1 / 2}\left(\Gamma_{3}\right)$ - пространство сужений на $\Gamma_{3}$ функций из $\widetilde{H}^{1 / 2}\left(\Gamma_{2,3}\right)$ с нормой inf и $\widehat{H}^{-1 / 2}\left(\Gamma_{3}\right)$ - пространство сужений на $\Gamma_{3}$ элементов из $\widetilde{H}^{-1 / 2}\left(\Gamma_{1,3}\right)$ с нормой inf. Далее, через $\mathscr{E}_{2}$ обозначим оператор продолжения функций из $\widehat{H}^{1 / 2}\left(\Gamma_{3}\right)$ через границу области $\Gamma_{2}$ до функций из 
$\widetilde{H}^{1 / 2}\left(\Gamma_{2,3}\right)$ и через $\mathscr{E}_{1}$ - оператор продолжения элементов из $\widehat{H}^{-1 / 2}\left(\Gamma_{3}\right)$ через границу области $\Gamma_{1}$ до элементов из $\widetilde{H}^{-1 / 2}\left(\Gamma_{1,3}\right)$. Эти два оператора получаются из известных нам операторов продолжения локализацией.

Предложение 7.1. Пространства $\widehat{H}^{-1 / 2}\left(\Gamma_{3}\right)$ и $\widehat{H}^{1 / 2}\left(\Gamma_{3}\right)$ дуалънъь относительно продолжения бормы $(\varphi, \psi)_{\Gamma_{3}}=\left(\mathscr{E}_{1} \varphi, \mathscr{E}_{2} \psi\right)_{\Gamma}$ на их прямое произведение.

Поясним, что на границе с областью $\Gamma_{1}$ пространство $\widehat{H}^{1 / 2}\left(\Gamma_{3}\right)$ локально «похоже» на $\widetilde{H}^{1 / 2}\left(\Gamma_{3}\right)$, а $\widehat{H}^{-1 / 2}\left(\Gamma_{3}\right)$ - на $H^{-1 / 2}\left(\Gamma_{3}\right)$, и на границе с $\Gamma_{2}$ пространство $\widehat{H}^{-1 / 2}\left(\Gamma_{3}\right)$ локально «похоже» на $\widetilde{H}^{-1 / 2}\left(\Gamma_{3}\right)$, а $\widehat{H}^{1 / 2}\left(\Gamma_{3}\right)-$ на $H^{1 / 2}\left(\Gamma_{3}\right)$. Поэтому на обеих частях границы области $\Gamma_{3}$ ситуация стандартна с точки зрения двойственности, хотя формально эти пространства немного нового типа.

Формула Грина сейчас имеет вид $\Phi_{\Omega}(u, v)=\left(T^{+} u, v^{+}\right)_{\Gamma_{3}}$. Из наших результатов в $\S \S 2-3$ следует, что решение в рассматриваемом классе однозначно определяется, если задать данные Дирихле в $\widehat{H}^{1 / 2}\left(\Gamma_{3}\right)$, при этом данные Неймана на $\Gamma_{3}$ попадают в $\widehat{H}^{-1 / 2}\left(\Gamma_{3}\right)$. Точно так же решение в нашем классе однозначно определяется, если задать данные Неймана в $\widehat{H}^{-1 / 2}\left(\Gamma_{3}\right)$, при этом данные Дирихле на $\Gamma_{3}$ попадают в $\widehat{H}^{1 / 2}\left(\Gamma_{3}\right)$. Поэтому определены два обратимых и взаимно обратных оператора

$$
D_{\Gamma_{3}}: \widehat{H}^{1 / 2}\left(\Gamma_{3}\right) \rightarrow \widehat{H}^{-1 / 2}\left(\Gamma_{3}\right), \quad N_{\Gamma_{3}}: \widehat{H}^{-1 / 2}\left(\Gamma_{3}\right) \rightarrow \widehat{H}^{1 / 2}\left(\Gamma_{3}\right),
$$

переводящие данные Дирихле на $\Gamma_{3}$ в данные Неймана на $\Gamma_{3}$ и обратно. На этих решениях мы имеем

$$
\Phi_{\Omega}(u, u)=\left(T^{+} u, N_{\Gamma_{3}} T^{+} u\right)_{\Gamma_{3}}=\left(D_{\Gamma_{3}} u^{+}, u^{+}\right)_{\Gamma_{3}} .
$$

Можно написать неравенства типа Гординга для наших операторов. Если $L=$ $\widetilde{L}$, то в $\widehat{H}^{-1 / 2}\left(\Gamma_{3}\right)$ можно ввести скалярное произведение $\langle\psi, \psi\rangle_{\Gamma_{3}}=\left(N_{\Gamma_{3}} \psi, \psi\right)_{\Gamma_{3}}$ и рассматривать $N_{\Gamma_{3}}$ как компактный самосопряженный оператор. Можно получить аналоги теорем 4.1 и 6.7. На деталях останавливаться не будем.

Два других варианта расположения области $\Gamma_{3}$ не сложнее, и останавливаться на них тоже не будем.

7.2. Третье граничное условие вместо условия Неймана. Вернемся к задаче (1.1)-(1.2), но заменим второе условие в (1.2) следующим:

$$
T^{+} u+\sigma u^{+}=h \quad \text { на } \Gamma_{2} \text {. }
$$

Здесь $\sigma(x)$ - заданная матрица из $L_{\infty}\left(\Gamma_{2}\right)$. Подобное граничное условие рассматривалось в очень многих работах, например, в [24] (случай $\Gamma_{2}=\Gamma$ ) и [34] (скалярное уравнение). Подробно мы рассматривать его не будем, на это потребовалось бы много места. Ограничимся следующими соображениями. Подставляя в формулу Грина $T^{+} u=h-\sigma u^{+}$, приходим к формуле

$$
\Phi_{\Omega}(u, v)+\left(\sigma u^{+}, v^{+}\right)_{\Gamma_{2}}=(L u, v)_{\Omega}+\left(h, v^{+}\right)_{\Gamma_{2}} .
$$

Теперь слева при $u=v$ мы имеем квадратичную форму

$$
\Phi_{\Omega}(u, u)+\left(\sigma u^{+}, u^{+}\right)_{\Gamma_{2}}
$$

и можем обобщать наши результаты, если она коэрцитивна в нашем смысле на $H^{1}(\Omega)$. Проще всего случай, когда $\operatorname{Re}\left(\sigma u^{+}, u^{+}\right)_{\Gamma_{2}} \geqslant 0$. Если же это условие не 
выполнено, то следует учесть, что в левую часть добавлено слагаемое меньшего порядка. Действительно, при сколь угодно малых $\alpha>0$ и $\beta>0$

$$
\begin{aligned}
\left|\left(\sigma u^{+}, u^{+}\right)_{\Gamma_{2}}\right| & \leqslant C_{1}\left\|u^{+}\right\|_{L_{2}(\Gamma)}^{2} \leqslant C_{1}\left\|u^{+}\right\|_{H^{\alpha}(\Gamma)}^{2} \leqslant C_{1}\|u\|_{H^{1 / 2+\alpha}(\Omega)}^{2} \\
& \leqslant \beta\|u\|_{H^{1}(\Omega)}^{2}+C_{\beta}\|u\|_{L_{2}(\Omega)}^{2} .
\end{aligned}
$$

Поэтому нужная коэрцитивность опять имеет место, если форма $\operatorname{Re}(c u, u)_{\Omega}$ достаточно велика.

7.3. Случай, когда граница разделена на несколько областей. Результаты заведомо можно обобщать на случай, когда поверхность разбита на конечное число областей конечным набором липшицевых $(n-2)$-мерных замкнутых поверхностей без самопересечений и без общих точек (предполагаем это из осторожности), в каждой из этих областей ставится или условие Дирихле, или условие Неймана, или спектральное условие. Ср. [34], [23] и [50].

Автор искренне благодарит Н. Д. Филонова и особенно Т. А. Суслину за обсуждение работы и ценные советы. Как отмечено в п. 6.3, теорему 6.8 мы получили с ней вместе.

Наш список литературы содержит далеко не все работы по смешанным задачам. Много дополнительных ссылок можно найти в работах этого списка.

\section{ЛитерАТУРА}

[1] M. S. Agranovich, On a mixed Poincaré-Steklov type spectral problem in a Lipschitz domain, Russian J. Math. Phys., 13:3 (2006), 239-244.

[2] M. S. Agranovich, Remarks on potential spaces and Besov spaces in a Lipschitz domain and on Whitney arrays on its boundary, Russian J. Math. Phys., 15:2 (2008), 146-155.

[3] М. С. Агранович, Операторь типа потенииала и задачи сопряжения для силъно эллиптических систем 2-го порядка в областях с липшищевой гранищей, Функц. анализ и его прил., 43:3 (2009), 3-25.

[4] М. С. Агранович, Сильно эллиптические системы 2-го порядка с граничными условиями на незамкнутой липшищевой поверхности, Функц. анализ и его прил., 45:1 (2011), 1-15.

[5] М. С. Агранович, Спектралъные задачи в липшищевых областях, Современная математика. Фундаментальные направления, 39 (2011) (в печати).

[6] М. С. Агранович, Б. А. Амосов, Оценки s-чисел и спектральные асимптотики для интегральных операторов типа потенииала на негладких поверхностях, Функц. анализ и его прил., 30:2 (1996), 1-18.

[7] М. Ш. Бирман, М. З. Соломяк, Асимптотика спектра вариационных задач на решениях эллиптических уравнений, Сиб. матем. журн., 20:1 (1979), 3-22.

[8] М. Ш. Бирман, М. З. Соломяк, Количественный анализ в теоремах вложения Соболева и приложения $\kappa$ спектральной теории, в кн.: «Десятая математическая школа», Инст. матем. АН УССР, Киев, 1974, 5-189.

[9] М. Ш. Бирман, М. С. Соломяк, Асимптотика спектра псевдодифференциальных операторов с анизотропно-однородными символами, Вестник ЛГУ, №13, матем. механ. астрон., вып. 3 (1977), 13-21.

[10] R. M. Brown, The mixed problem for Laplace's equation in a class of Lipschitz domains, Comm. Partial Differential Equations, 19:7-8 (1994), 1217-1233.

[11] R. M. Brown, I. Mitrea, The mixed problem for the Lamé system in a class of Lipschitz domains, J. Differential Equations, 246:7 (2009), 2577-2589. 
[12] M. Costabel, Boundary integral operators in Lipschitz domains: elementary results, SIAM J. Math. Anal., 19:3 (1988), 613-626.

[13] Р. Курант, Д. Гильберт, Методъ математической физики, m. I, ГТТИ, М., 1934.

[14] B. E. J. Dahlberg, C. E. Kenig, G. C. Verchota, Boundary value problems for the system of elastostatics in Lipschitz domains, Duke Math. J., 57:3 (1988), 795-818.

[15] Г. И. Эскин, Краевые задачи для эллиптических псевдодифференциальных уравнений, Наука, М., 1973.

[16] V. I. Fabrikant, Mixed Boundary Value Problems of Potential Theory and their Applications in Engineering, Kluwer, Dorderecht, 1991.

[17] J. A. Griepentrog, K. Gröger, H.-Chr. Kaiser, J. Rehrberg, Interpolation for function spaces related to mixed boundary value problems, Math. Nachr., 241 (2002), 110-120.

[18] J. A. Griepentrog, H.-Chr. Kaiser, J. Rehrberg, Heat kernel and resolvent properties for second order elliptic differential operators with general boundary conditions on $L_{p}$, Adv. Math. Sci. Appl., 11 (2001), 87-112.

[19] K. Gröger, $A W^{1, p}$-estimate for solutions to mixed boundary value problems for second order elliptic differential equations, Math. Ann., 283:4 (1989), 679-687.

[20] G. C. Hsiao, W. L. Wendland, Boundary Integral Equations, Springer-Verlag, Berlin, 2008.

[21] D. Jerison, C. E. Kenig, The inhomogeneous Dirichlet problem in Lipschitz domains, J. Funct. Anal., 130:1 (1995), 161-219.

[22] A. Jonsson, H. Wallin, Function Spaces on Subsets of $\mathbb{R}^{n}$, Harwood Academic Publishers, 1984.

[23] В. И. Лебедев, В. И. Агошков, Операторы Пуанкаре-Стеклова и их приложения в анализе, Отдел вычислительной математики АН CССР, М., 1983.

[24] W. McLean, Strongly Elliptic Systems and Boundary Integral Equations, Cambridge Univ. Press, Cambridge, 2000.

[25] G. Métivier, Valeurs propres de problémes aux limites elliptiques irreguliers, Bull. Soc. Math. France, Mémoire 51-52 (1977), 125-219.

[26] S. E. Mikhailov, Traces, extensions, co-normal derivatives and solution regularity of elliptic systems with smooth and non-smooth coefficients, http://arxiv.org/abs/ $0906.3875 \mathrm{v} 1$.

[27] I. Mitrea, M. Mitrea, The Poisson problem with mixed boundary conditions in Sobolev and Besov spaces in non-smooth domains, Trans. Amer. Math. Soc., 359:9 (2007), 4143-4182.

[28] M. Mitrea, M. Taylor, Boundary layer methods for Lipschitz domains in Riemannian manifolds, J. Funct. Anal., 163:2 (1999), 181-251.

[29] M. Mitrea, M. Taylor, Potential theory on Lipschitz domains in Riemannian manifolds: Sobolev-Besov space results and the Poisson problem, J. Funct. Anal., 176:1 (2000), 1-79.

[30] Д. Г. Натрошвили, О. О. Чкадуа, Е. М. Шаргородский, Смешаннъе задачи для однородных анизотропных упругих сред, Труды Института прикладной математики им. Векуа, 39 (1990), 133-178.

[31] J. Nečas, Les méthodes directes en théorie des équations elliptiques, Masson, Paris, 1967.

[32] O. A. Oleinik, A. S. Shamaev, G. A. Yosifian, Mathematical Problems in Elasticity and Homogenization, North Holland, Amsterdam, 1992.

[33] K. A. Ott, R. M. Brown, The mixed problem for the Laplacian in Lipschitz domains http://arxiv.org/abs/0909.0061v2.

[34] Б. В. Пальцев, О смешанной задаче с неоднородными граничными условиями для эллиптических с параметром уравнений второго порядка в липшищевых областлх, Матем. сб., 187:4 (1996), 59-116. 
[35] T. von Petersdorff, Boundary integral equations for mixed Dirichlet, Neumann and transmission problems, Math. Methods Appl. Sci., 11:2 (1989), 183-213.

[36] G. Rozenblum, G. Tashchiyan, Eigenvalue asymptotics for potential type operators on Lipschitz surfaces, Russian J. Math. Phys., 13:3 (2006), 326-339.

[37] V. S. Rychkov, On restrictions and extensions of the Besov and Triebel-Lizorkin spaces with respect to Lipschitz domains, J. London Math. Soc. (2), 60:1 (1999), 237-257.

[38] G. Savaré, Regularity and perturbation results for mixed second order elliptic problems, Comm. Partial Differential Equations, 22:5-6 (1997), 869-899.

[39] E. Shamir, Regularization of mixed second-order elliptic equations, Israel J. Math., 6 (1968), 150-168.

[40] И. Я. Шнейберг, Спектральные свойства линейных операторов в интерполяиионных семействах банаховых пространств, Матем. исслед., 9:2 (1974), 214-227.

[41] I. N. Sneddon, Mixed Boundary Value Problems in Potential Theory, Elsevier, New York, 1966.

[42] E. Stephan, Boundary integral equations for mixed boundary value problems, screen and transmission problems in $\mathbb{R}^{3}$, Habilitationsschrift, Darmstadt, 1984 (THDpreprint 848).

[43] E. P. Stephan, Boundary integral equations for mixed boundary value problems in $\mathbb{R}^{3}$, Math. Nachr., 134 (1987), 21-53.

[44] Т. А. Суслина, Асимптотика спектра вариационных задач на решениях однородного эллиптического уравнения при наличии связей на части границы, в кн.: Проблемы мат. анализа, т. 9, ЛГУ, 1984, 84-97.

[45] T. A. Suslina, Spectral asymptotics of variational problems with elliptic constraints in domains with piecewise smooth boundary, Russian J. Math. Phys., 6:2 (1999), 214-234.

[46] J. D. Sykes, R. M. Brown, The mixed boundary problem in $L^{p}$ an Hardy spaces for Laplace's equation on a Lipschitz domain, in: Contemporary Mathematics, vol. 227, Amer. Math. Soc., 2001, 1-18.

[47] H. Triebel, Function spaces in Lipschitz domains and on Lipschitz manifolds. Characteristic functions as pointwise multipliers, Rev. Mat. Complut., 15:2 (2002), 475-524.

[48] G. Uhlmann, Inverse boundary problems and applications, Astérisque, 207 (1992), 153-207.

[49] G. Verchota, Layer potentials and regularity for the Dirichlet problem for Laplace's equation in Lipschitz domains, J. Funct. Anal., 59:3 (1984), 572-611.

[50] В. И. Войтицкий, Н. Д. Копачевский, П. А. Старков, Многокомпонентные задачи сопряжения и вспомогательные абстрактные краевые задачи, Современная математика. Фундаментальные направления, 34 (2009), 5-44.

Московский институт электроники и математики

Поступило в редакцию e-mail: magran@orc.ru 16 декабря 2010 г. 